\title{
MedienPädagogik
}

Zeitschrift für Theorie und Praxis der Medienbildung

Themenheft Nr. 28: Tagungsband: Bildung gemeinsam verändern: Diskussionsbeiträge und Impulse aus Forschung und Praxis. Herausgegeben von David Meinhard, Valentin Dander, Andrea Gumpert, Christoph Rensing, Klaus Rummler und Timo van Treeck.

\section{Gemeinsam hilfreich oder einsam lästig? Beurteilung von praktikumsbegleitenden Weblogs von angehenden Lehr- personen}

Nives Egger

\begin{abstract}
Zusammenfassung
Weblogs werden an Hochschulen vermehrt zur Unterstützung von Lern- und Reflexionsprozessen eingesetzt, in der Lehrer/innenbildung auch zunehmend während der berufspraktischen Ausbildung. Die Studierenden beurteilen den Einsatz von Weblogs jedoch unterschiedlich. Dabei ist wenig bekannt, welchen Einfluss die Vergabe und das Erhalten von Peerfeedback auf den Beurteilungsprozess von praktikumsbegleitenden Weblogs sowie auf den Umfang von Blogpostings haben. Im folgenden Beitrag wird dieser Frage mittels einer Befragung von 74 angehenden Lehrpersonen nachgegangen, die praktikumsbegleitende Weblogs mit oder ohne Peerfeedback nutzten, um über herausfordernde Ereignisse im Praktikum zu reflektieren. Die Ergebnisse zeigen, dass Studierende, die Peerfeedback erhielten und andere Blogbeiträge kommentierten, den Weblogeinsatz nützlicher einschätzen sowie eine höhere Motivation und positivere Einstellung gegenüber den Einsatz von Weblogs aufweisen als Studierende, die ohne Peerfeedback bloggen. Keinen Einfluss hat Peerfeedback auf den Umfang der Blogbeiträge und auf die dafür aufgewendete Zeit.
\end{abstract}

Productive cooperation or solitary nuisance? How pre-service teachers assess the usefulness of internship-accompanying weblogs.

\begin{abstract}
Weblogs are being increasingly used in higher education to support the learning and reflection processes of students. In teacher training, they are often employed in school internships or practical training phases. Students assess the usefulness of weblogs differently. However, little is known regarding the influence of peer feedback on the quantity and quality of blog postings and the perceived value of weblogs for students. In the present study, a sample of 74 students enrolled in teacher preparation programs used weblogs during a 4-week school internship to write about and reflect on stressful situations experienced during classroom teaching - one group with, and one without peer feedback. The results show that students who had received peer feedback and had commented on other blog posts themselves, judged weblog use to be more valuable and
\end{abstract}


reported a more positive attitude and a higher motivation towards using them. However, no difference was found between groups with and without peer feedback regarding the length of blog postings or the time spent on writing them.

\section{Einleitung}

Unterrichtspraktika stellen für angehende Lehrpersonen ein zentrales Ausbildungsfeld zum Erwerb von berufsspezifischen Kompetenzen dar (vgl. Hascher 2012, 1). Jedoch zeigen empirische Studien, dass der Übergang von der theoretischen Hochschulausbildung ins Schulfeld für die Studierenden ein hohes Belastungspotenzial mit sich bringen kann, weil sie mit einer Vielzahl von Herausforderungen (wie Klassenführung, Unterrichtsvorbereitung, eigene Unsicherheiten in Bezug auf die Unterrichtstätigkeit) konfrontiert werden (vgl. Gardner 2010, 23). Eine differenzierte Reflexion solcher Herausforderungen kann helfen, besser mit Belastungen im Praktikum umgehen zu können. Es stellt sich dabei die Frage, wie solche reflexiven Prozesse sinnvollerweise angeleitet werden können. Als eine bewährte Massnahme gelten persönliche Lerntagebücher oder Portfolios. In der Lehrer/innenbildung hat sich der Einsatz von persönlichen Lerntagebüchern oder Portfolios zur Förderung von Reflexionsprozessen etabliert und gilt mehrheitlich als ein erfolgsversprechendes Mittel (vgl. Gläser-Zikuda und Hascher 2007). Durch die zunehmende Digitalisierung und den Einfluss neuer Medien stellt der Einsatz von digitalen Tagebüchern eine konsequente Weiterentwicklung bisheriger Reflexionsinstrumente mit zusätzlichen Möglichkeiten dar. Der öffentliche und digitale Charakter von Weblogs ermöglicht eine unmittelbare Kommunikation und Unterstützung durch Peers und Mentoren/-innen in Form von Kommentaren. Bislang wenig erprobt sind jedoch praktikumsbegleitende Weblogs als Methode zur Bewältigung von herausfordernden Ereignissen bei angehenden Lehrpersonen. Dieser Ansatz wird im Folgenden vorgestellt. Dabei wird untersucht, ob Peerfeedback einen Einfluss auf den Umfang und den Zeitaufwand für das Verfassen eines Beitrages sowie auf die selbsteingeschätzte Nützlichkeit, Motivation und Einstellung von Nutzerinnen und Nutzern praktikumsbegleitender Weblogs hat.

\section{Einsatzformen von praktikumsbegleitenden Weblogs}

Überblicksartikel zeigen, dass Weblogs im Hochschulkontext zur Förderung von Reflexions- und Lernprozessen intensiv genutzt werden (vgl. Grell und Rau 2011; Sim und Hew 2010). In den letzten Jahren wurden Weblogs auch vermehrt in der berufspraktischen Ausbildung eingesetzt. Dabei konnte die Effektivität von Weblogs als Instrument zum Informationsaustausch, zur Generierung von Problemlösestrategien sowie zur Unterstützung von Reflexionsprozessen in mehreren Studien bestätigt 
werden (vgl. Harland und Wondra 2011, 131; Wopereis et al. 2010, 254). Besondere Potenziale von Weblogs liegen im Bereich der emotionalen Unterstützung (vgl. Chu, Kwan und Warning 2012, 172; Deng und Yuen 2007, 198). Studien aus therapeutischen Kontexten liefern zudem Befunde, dass Bloggen den Verarbeitungsprozess von belastenden Ereignissen fördern kann (vgl. Petko et al. 2015). Ein wesentlicher Vorteil von Weblogs liegt dabei in der Kommentarfunktion. Zum Einfluss von Peerfeedback finden sich in der Literatur mehrheitlich positive Befunde. Beispielweise hat Peerfeedback einen positiven Effekt auf die Qualität der Schreibergebnisse (vgl. Chen et al. 2011, 2; Nicolaidou 2013, 409) und führt insgesamt zu besseren Lernergebnissen (vgl. Novakovich 2016, 10). Auch findet sich ein positiver Zusammenhang zwischen der Anzahl an Kommentaren und Blogbeiträgen (vgl. Bernhardt und Wolf 2012, 148). Trotzdem kann das Bloggen für eine Leserschaft sich auch negativ auswirken, wie die Studie von Xie, Ke und Sharma (vgl. 2008, 22) zeigt. Blogpostings von Studierenden, die von ihren Peers kommentiert wurden, wiesen ein geringeres Reflexionsniveau auf als Beiträge von Studierenden, die nicht durch andere kommentiert wurden. Möglichweise werden Inhalte weniger tiefgründig präsentiert, wenn Postings einen öffentlichen Charakter haben (vgl. ebd., 23). Ob Weblogs und deren Potenziale zielführend genutzt werden, hängt auch davon ab, ob solche Schreibprozesse instruktional unterstützt werden. Konkrete Schreibaufträge oder Prompts begünstigen Reflexionsprozesse und metakognitive Strategien (vgl. Kori et al. 2014, 48; Petko et al. 2014, 10). Daher ist es naheliegend, dass auch die Vergabe von Kommentaren durch Schreibaufträge oder Prompts gesteuert wird. Die Technologieakzeptanzforschung geht davon aus, dass die tatsächliche Nutzung von Technologien massgeblich von der Akzeptanz und der Einstellung des Nutzers gegenüber der Technologie abhängt. Dabei spielt vor allem die wahrgenommene Nützlichkeit eine entscheidende Rolle (Venkatesh et al. 2012, 163). Aber auch die allgemeine Einstellung gegenüber der Technologie und der erwartete Spassfaktor sind zentrale Prädiktoren für die Nutzung (ebd., 164). Bisher gibt es nur wenige Erkenntnisse über die Rolle von Peerfeedback hinsichtlich ihrer Nützlichkeitsbeurteilung, Motivation und Einstellung der Studierenden zu praktikumsbegleitenden Weblogs. Wenig bekannt ist auch, welchen Einfluss das Fehlen von Kommentaren auf den Umfang der Blogbeiträge hat.

Basierend auf den theoretischen Darlegungen und den empirischen Desideraten aus der empirischen Bildungsforschung werden die folgenden zwei Fragestellungen abgeleitet:

1. Zeigen sich Unterschiede zwischen Studierenden, die mit und ohne Peerfeedback bloggen hinsichtlich ihrer Bloggingaktivitäten (Umfang und Zeitaufwand)?

2. Zeigen sich Unterschiede zwischen den Studierenden mit und ohne Peerfeedback hinsichtlich ihrer Einschätzung von Nützlichkeit, Motivation und ihrer Einstellung zu praktikumsbegleitenden Weblogs? 


\section{Einsatzszenario und Stichprobe}

Bei der vorliegenden Studie handelt es sich um eine vom Schweizerischen Nationalfonds (SNF) geförderte Studie «Blogging in internships: Writing online journals as a method for coping with stress in medical and teacher education»(Nr. 100013_146054). An der Studie waren neben der Pädagogischen Hochschule Schwyz (PHSZ) die Pädagogische Hochschule Zürich (PH Zürich) sowie das Institut für Medizinische Lehre (IML) der Universität Bern beteiligt. Aus dieser Studie sind bereits einige Publikationen entstanden, die auch in die vorliegende Publikation einflossen.

Im Rahmen einer quasi-experimentellen Studie wurden alle Studierenden des zweiten Studienjahres der Pädagogischen Hochschule Schwyz (CH), die im Juni 2014 und im Januar 2015 ihr Fachpraktikum absolvierten, randomisiert in vier Experimentalgruppen und eine Kontrollgruppe unterteilt. Die Experimentalgruppen bloggten täglich über Herausforderungen während ihres vierwöchigen Praktikums. Die Studierenden der Kontrollgruppe bloggten nicht, sondern schrieben am Ende des Praktikums einen mehrseitigen Abschlussbericht. In der Experimentalgruppe wurden verschiedene Bedingungen variiert. Einerseits wurden unterschiedliche Schreibaufträge vergeben und andererseits bloggten die Studierenden mit oder ohne Peerfeedback. Studierende, die mit Peerfeedback bloggten, bekamen den Auftrag, täglich einen Beitrag ihrer Mitstudierenden zu lesen und zu kommentieren. Die Hälfte der Experimentalgruppe erhielt den Schreibauftrag, herausfordernde Ereignisse durch die Übernahme einer positiven Sicht- bzw. Denkweise zu reflektieren, die andere Hälfte wurde dazu aufgefordert, konkrete Problemlösestrategien zu generieren. Auch die Kommentarvergabe wurde durch Schreibaufträge gesteuert. Alle Studierenden besuchten vor dem Praktikum einen Workshop, in dem ihnen Strategien zum Umgang mit belastenden Situationen vermittelt wurden. Der Workshop basiert auf dem Trainingsmanual von Kaluza (vgl. 2011). Als Blogging-Plattform wurde «blogpraktikum. ch» verwendet, das am Institut für Medien und Schule (IMS) an der Pädagogischen Hochschule Schwyz entwickelt wurde (vgl. Petko 2013).

Die Datenerhebung erfolgte in zwei Kohorten und umfasste zwei Studierendenjahrgänge. Für die vorliegende Studie werden als empirische Grundlage nur die Daten der 2. Kohorte der Experimentalgruppe (Fachpraktikum mit Start im Januar 2015) verwendet, da einige verwendete Items nur bei dieser Studierendenkohorte erhoben wurde. Diese Stichprobe umfasst $\mathrm{N}=74$ Studierende im Alter von 19 bis 40 Jahren $(M=23.61, S D=3.61) .84$ Prozent sind weibliche und 16 Prozent sind männliche Studierende. Davon haben $n=36$ Studierende Peerfeedback erhalten, die Gruppe ohne Peerfeedback bestand aus $n=38$ Studierenden. 


\section{Erhebungsinstrumente}

Unmittelbar nach dem Praktikum wurden die Studierenden mit einem standardisierten Onlinefragebogen zum Einsatz von praktikumsbegleitenden Weblogs befragt. Neben dem selbsteingeschätzten Zeitaufwand für das Verfassen eines Postings wurde auch die persönliche Einschätzung bezüglich der Nützlichkeit von Weblogs als Methode zur Stressreduktion, die Motivation zu bloggen sowie die Einstellung der Studierenden gegenüber praktikumsbegleitenden Weblogs erhoben. Als Instrumente wurden standardisierte, aber auch eigens entwickelte Items verwendet, die im Folgenden beschrieben werden:

- Blogaktivitäten: Die Bloggingaktivitäten der Studierenden wurden einerseits anhand der Zeichenanzahl pro Blogposting und andererseits mit dem durchschnittlichen Zeitaufwand für das Schreiben eines Blogbeitrages ermittelt («Wie viele Minuten haben Sie normalerweise täglich aufgewendet für das Schreiben eines Blogbeitrags?»).

- Die Motivation von praktikumsbegleitenden Weblogs wurde mit der Skala «Freude am Schreiben von Blogbeiträgen», 2 Items, Cronbach's alpha=.78 (Beispielitem: «Im Grossen und Ganzen habe ich meine Blogbeiträge gerne geschrieben») erfasst. Das Antwortformat war fünfstufig.

- Die Nützlichkeit wurde mit dem Item «Das Bloggen betrachte ich als hilfreiche Methode, um besser mit Belastungen während des Praktikums umzugehen» erfasst. Das Antwortformat war fünfstufig.

- Zur Erfassung der Einstellung der Studierenden gegenüber dem Einsatz von Weblogs während des Praktikums wurde die Subskala «Attitude toward using technology» (4 Items, Cronbach's alpha=.87) des Fragebogens «Unified Theory of Acceptance and Use of Technology (UTAUT)» von Venkatesh et al. (2003) verwendet. (Beispielitem: «Ich arbeitete gerne mit dem Weblog während des Praktikums») Das Antwortformat war siebenstufig.

Die erwähnten Items bzw. Skalen wurden unmittelbar nach Abschluss des Praktikums erhoben.

\section{Auswertungsverfahren}

Die Datenauswertung beruht auf deskriptiven Analysen. Der Vergleich zwischen Studierenden, die mit und solchen, die ohne Peerfeedback bloggten, wurde mittels nonparametrischen Verfahren (Mann-Whitney-U-Tests) auf signifikante Unterschiede geprüft $(p<.05)$. Dieses Verfahren wurde aufgrund der kleinen Fallzahlen gewählt. Alle Auswertungen erfolgten mit R 3.1.1 sowie den Packages sjPlot(), psych(), $\operatorname{car}()$ und ggPlot2(). 


\section{Ergebnisse}

Im Folgenden werden die Ergebnisse der Studierendenbefragung zu Bloggingaktivitäten sowie selbst eingeschätzte Nützlichkeit, Motivation und Einstellung gegenüber dem Einsatz von praktikumsbegleitenden Weblogs im Vergleich von Studierenden, die mit und ohne Peerfeedback gebloggt haben, dargestellt.

\section{Bloggingaktivitäten der Studierenden mit und ohne Peerfeedback}

Die Studierenden, die auf ihre Blogbeiträge Kommentare erhielten, haben angegeben, dass sie durchschnittlich $M=15.61$ ( $S D=8.75$ ) Minuten gebraucht haben, um ein Blogposting zu verfassen. Studierende, die keine Kommentare erhielten, haben durchschnittlich $M=14.08$ ( $S D=5.18$ ) Minuten aufgewendet. Es zeigt sich hier kein signifikanter Unterschied zwischen den Gruppen. Auch bei der Anzahl der Zeichen pro Blogposting unterscheiden sich die beiden Gruppen nicht voneinander. Studierende, die mit Peerfeedback bloggten, verwendeten kaum mehr Zeichen pro Blogposting $(M=1434.63, S D=403.58)$ als Studierende ohne Peerfeedback $(M=1458.38, S D=474.87)$.

\section{Selbsteingeschätze Nützlichkeit und Motivation der Studierenden gegenüber dem zum} Einsatz von praktikumsbegleitenden Weblogs

Generell zeigt die Analyse, dass Studierende praktikumsbegleitende Weblogs eher nicht als nützlich erachten, um besser mit Belastungen umzugehen (vgl. Abb. 1, fünfstufige Likertskala). Jedoch zeigt sich, dass Studierende, die auf ihre Beiträge Kommentare erhielten, das Bloggen als nützlicher einschätzen ( $M=2.33, S D=1.01)$ als Studierende ohne Kommentarfunktion ( $M=1.84, S D=.97)$. Es zeigt sich hier ein signifikanter Unterschied ( $U=493.00, p=.030)$. 


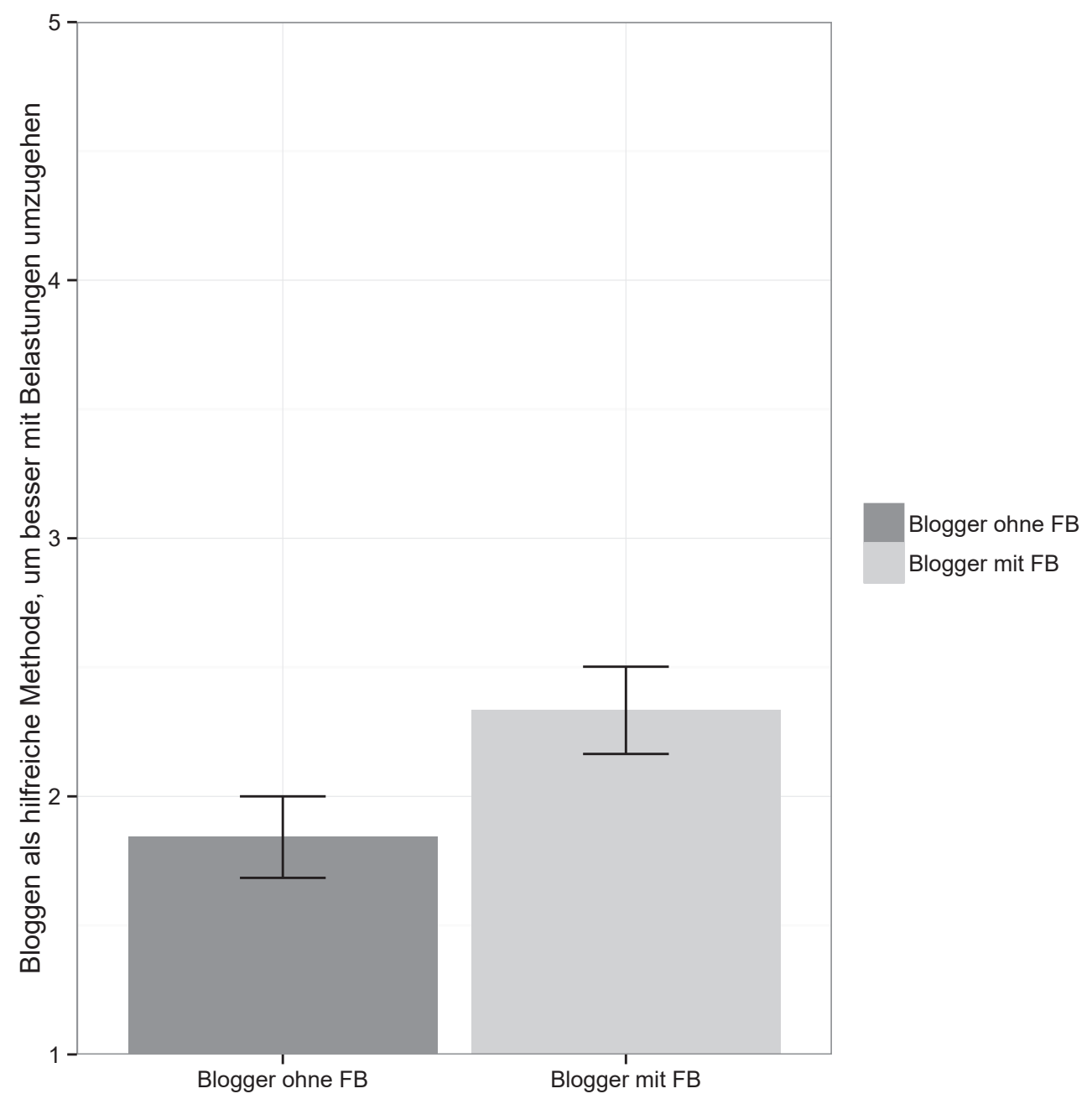

Abb. 1.: Nützlichkeit von praktikumsbegleitenden Weblogs im Vergleich zwischen Studierenden mit $(n=36)$ und ohne Peerfeedback $(n=38)$.

Im Folgenden wurde die Motivation der Studierenden zum Einsatz von praktikumsbegleitenden Weblogs ermittelt. Dafür wurden die Studierenden gefragt, ob ihnen das Bloggen Freunde bereitet hat. Auch hier zeigt sich generell, dass den Studierenden das Bloggen wenig Freunde machte (vgl. Abb. 2, fünfstufige Likertskala). Jedoch machte den Studierende, die mit Feedback bloggten ( $M=2.73, S D=.86)$ das Verfassen von Postings mehr Freude als Studierenden, die keine Kommentare von ihren Mitstudierenden erhielten und keine anderen Beiträge lesen konnten ( $M=2.07, S D=.95)$. Dieser Unterschied ist signifikant $(U=409.00, p=.003)$. 


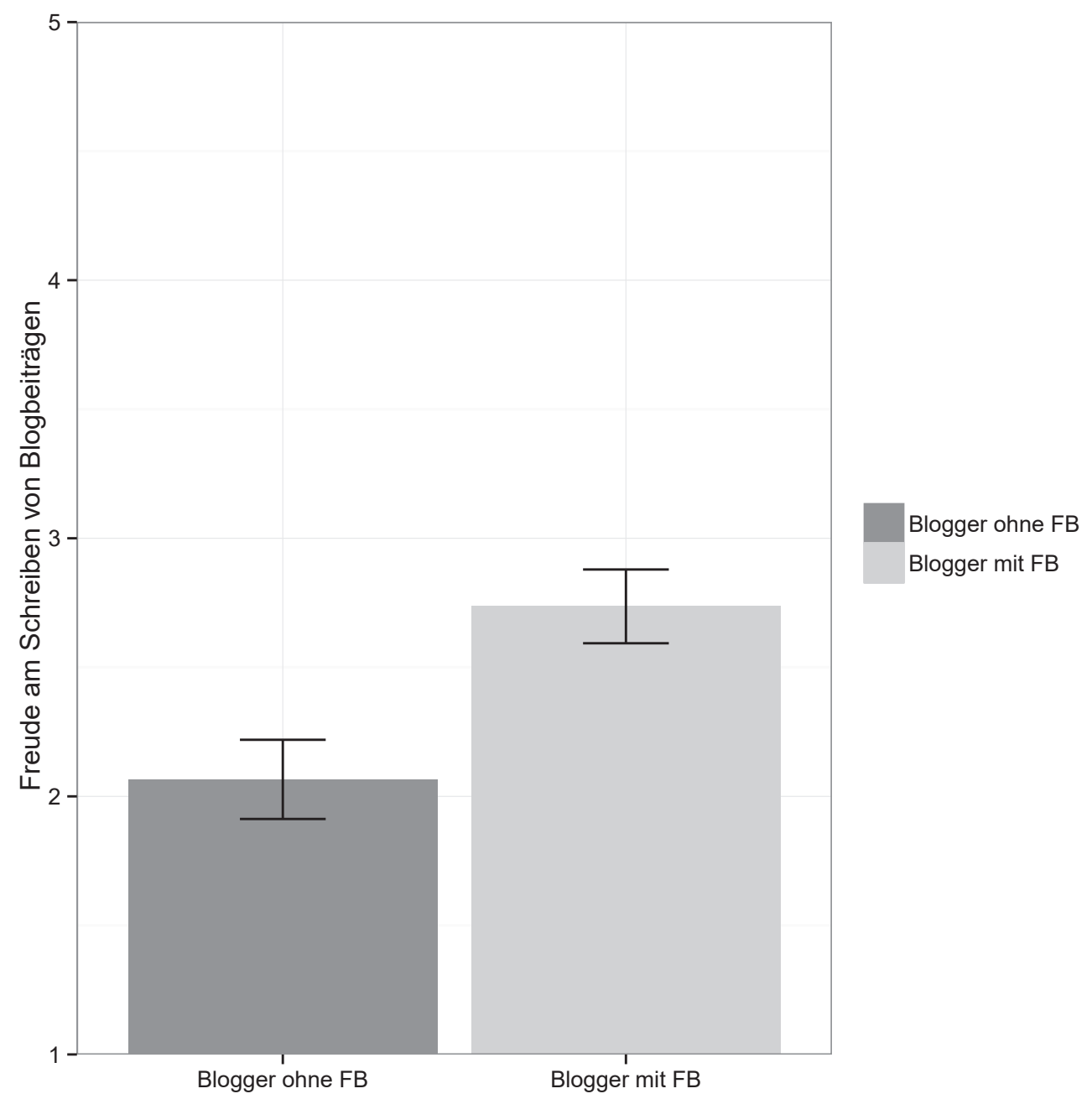

Abb. 2.: Motivation der Studierenden zum Einsatz von praktikumsbegleitenden Weblogs im Vergleich zwischen Studierenden mit ( $n=36)$ und ohne Peerfeedback $(n=38)$.

\section{Einstellung der Studierenden gegenüber dem Einsatz von praktikumsbegleitenden Weblogs}

Tendenziell zeigt sich, dass Studierende eine eher ablehnende Einstellung gegenüber dem Einsatz von praktikumsbegleitenden Weblogs haben (vgl. Abb. 3, siebenstufige Likertskala). Eine positivere Einstellung weisen wiederum die Studierenden auf, die mit Kommentarfunktion bloggten $(M=3.30, S D=1.26)$. Auch hier zeigt sich ein signifikanter Unterschied $(U=457.50, p=.014)$ zu Studierenden, deren Blogs nicht kommentiert wurden $(\mathrm{M}=2.55, \mathrm{SD}=1.32)$. 


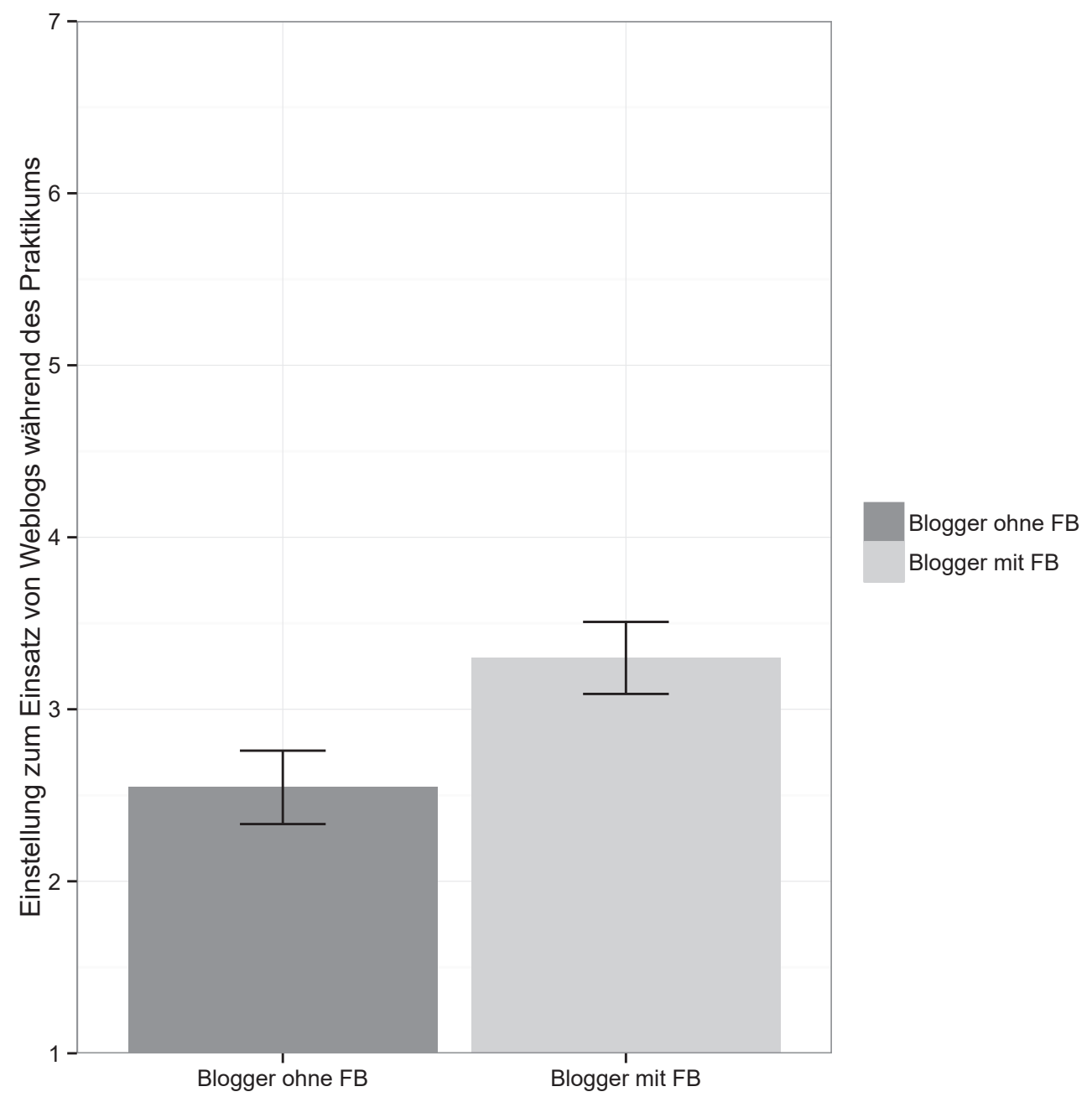

Abb. 3.: Einstellung zum Einsatz von praktikumsbegleitenden Weblogs im Vergleich zwischen Studierenden mit $(n=36)$ und ohne Peerfeedback $(n=38)$.

\section{Zusammenfassung und Diskussion}

Insgesamt zeigen die Befunde, dass Studierende tendenziell eher eine negative Einstellung gegenüber dem Einsatz von praktikumsbegleitenden Weblogs zur Reflexion von belastenden Ereignissen haben. Auch die Nützlichkeit wurde eher kritisch beurteilt. Die Vergleichsanalysen zeigen allerdings, dass der Einsatz von Weblogs im Praktikum signifikant besser beurteilt wird, wenn die Studierenden auf ihre Blogbeiträge Kommentare erhielten und die Möglichkeit hatten, andere Blogbeiträge zu lesen und zu kommentieren. Keinen Einfluss hatte Peerfeedback hingegen auf die Bloggingaktivitäten, d. h. die Studierenden wendeten nicht mehr Zeit auf, um einen Blogbeitrag zu verfassen und auch die Länge der Beiträge fiel nicht umfangreicher aus. Die gerin- 
ge Nützlichkeitsbeurteilung von praktikumsbegleitenden Weblogs könnte sich unter Umständen auf eine tendenziell eher geringe Stressbelastung während des Praktikums zurückführen lassen (vgl. Egger et al. 2016, 201). Einen weiteren Erklärungsansatz könnte auch der verpflichtende Charakter des Bloggens liefern. Aus früheren Untersuchungen ist bekannt, dass verordnetes Bloggen bei den Studierenden eher auf Ablehnung stösst (vgl. Hobbs 2007, 412). Jedoch darf daraus nicht geschlussfolgert werden, dass Bloggen auf freiwilliger Basis auf höhere Akzeptanz stösst. Eine weniger kritische Haltung zeigt sich eher dann, wenn das Bloggen andere Leistungsnachweise im Praktikum ersetzt und nicht ergänzt (vgl. Totter et al. 2016, 209). Hingegen wird die Bedeutung von Peerunterstützung als motivationsförderlicher Faktor beim Bloggen in der vorliegenden Studie bestätigt. Dieser Befund steht im Einklang mit der Selbstbestimmungstheorie, die die soziale Eingebundenheit als eine wesentliche Bedingung für die intrinsische Motivation sieht (vgl. Ryan und Deci 2000, 64). Es ist anzunehmen, dass die Studierenden die Nützlichkeit von praktikumsbegleitenden Weblogs primär nicht darin sehen, über belastende Ereignisse im Praktikum zu reflektieren, sondern vielmehr sich darüber auszutauschen. Dafür stellen digitale Tagebücher eine geeignete Austauschplattform dar, da sie jederzeit verfügbar und öffentlich zugänglich sind. Auch wenn Studierende praktikumsbegleitende Weblogs als nützlicher beurteilen, wenn ein sozialer Austausch stattfindet, stellt sich zukünftig die Frage, welchen Einfluss Peerfeedback auf die Reflexions- und Lernergebnisse hat. Dafür bedarf es noch weiterführender Analysen. Weiter stellt sich die Frage, wie Feedback hinsichtlich Häufigkeit und Öffentlichkeit gestaltet werden muss. Auch muss geklärt werden, wie stark die Rückmeldungen instruktional vorgegeben werden sollen, damit Studierende davon profitieren. Obwohl die Ergebnisse auf einer kleinen Stichprobe basieren und dadurch nicht generalisierbar sind und unter den speziellen Bedingungen - verpflichtend und täglich - interpretiert werden müssen, so zeigt sich doch, dass für die Studierenden das «gemeinsame Bloggen» einen Mehrwert gegenüber dem «einsamen Reflektieren» hat.

\section{Literatur}

Bernhardt, Thomas, und Karsten D. Wolf. 2012. «Akzeptanz und Nutzungsintensität von Blogs als Lernmedium in Onlinekursen.» In Digitale Medien - Werkzeuge für exzellente Forschung und Lehre, herausgegeben von Gottfried Csanyi, Franz Reichl, und Andreas Steiner, 141152. Münster, New York und München, Berlin: Waxmann.

Chen, Yu Liang, Eric Zhi Feng Liu, Ru Chu Shih, Chin Tsung Wu, und Shyan Ming Yuan 2011. «Use of peer feedback to enhance elementary students' writing through blogging.» British Journal of Educational Technology 42 (1): E1-E4. doi:10.1111/j.1467-8535.2010.01139.x. 
Chu, Samuel. K. W., Alvin. C. M. Kwan, und Peter Warning. 2012. «Blogging for Information Management, Learning, and Social Support during Internship.» Educational Technology \& Society 15 (2): 168-178.

Deng, Liping and Allan H. K. Yuen. 2007. «Exploring the role of weblogs in supporting learning communities: An integrative approach.» In ICT: Providing choices for learners and learning. Proceedings ascilite Singapore 2007. http://citeseerx.ist.psu.edu/viewdoc/download?doi= 10.1.1.91.9543\&rep=rep1\&type=pdf.

Egger, Nives, Alexandra Totter, und Dominik Petko. 2016. "Praktikumsbegleitende Weblogs: Ein geeignetes Mittel im Umgang mit Stress?» Zeitschrift für Hochschulentwicklung 11 (1):189-208. doi:10.3217/zfhe-11-01/11.

Gardner, Sallie. 2010. «Stress among prospective teachers: A review of the literature.» Australian Journal of Teacher Education 35 (8):18-28.

Gläser-Zikuda, Michaela, und Tina Hascher. 2007. «Zum Potential von Lerntagebuch und Portfolio. " In Lernprozesse dokumentieren, reflektieren und beurteilen. Lerntagebuch und Portfolio in Bildungsforschung und Bildungspraxis, herausgegeben von Michaela Gläser-Zikuda und Tina Hascher, 9-21. Bad Heilbrunn: Klinkhardt.

Grell, Petra, und Franco Rau. 2011. «Partizipationslücken - Social Software in der Hochschullehre.» MedienPädagogik (21):1-23. doi:10.21240/mpaed/21/2011.11.21.X.

Harland, Darci J., und Joshua. D. Wondra. 2011. «Preservice teachers' reflection on clinical experiences: A comparison of blog and final paper assignments.» Journal of Digital Learning in Teacher Education 27 (4):128-133. doi:10.1080/21532974.2011.10784669.

Hascher, Tina. 2012. «Lernfeld Praktikum-Evidenzbasierte Entwicklungen in der Lehrer/innenbildung.» Zeitschrift für Bildungsforschung 2 (2): 109-129. doi:10.1007/s35834-012-0032-6.

Hobbs, Valerie. 2007. «Faking it or hating it: can reflective practice be forced?» Reflective practice 8 (3): 405-417. doi:10.1080/14623940701425063.

Hoyt, Tim, und Monisha Pasupathi. 2008. «Blogging about trauma: Linguistic markers of apparent recovery.» E-Journal of Applied Psychology 4 (2):56-62. doi:10.7790/ejap.v4i2.106.

Kaluza, Gerd. 2011. Stressbewältigung. Trainingsmanual zur psychologischen Gesundheitsförderung (2. vollst. überarbeitete Auflage). Berlin, Heidelberg: Springer-Verlag.

Kori, Külli, Margus Pedaste, Äli Leijen, und Mario Mäeots. 2014. "Supporting reflection in technology-enhanced learning.» Educational Research Review 11:45-55. doi:10.1016/j.edurev.2013.11.003.

Nicolaidou, Iolie. 2013. «E-portfolios supporting primary students' writing performance and peer feedback. » Computers \& Education 68:404-415. doi:10.1016/j.compedu.2013.06.004.

Novakovich, Josip. 2016. "Fostering critical thinking and reflection through blog-mediated peer feedback». Journal of Computer Assisted Learning 32 (1):16-30. doi:10.1111/jcal.12114.

Pennebacker, James W. 1997. «Writing About Emotional Experiences as a Therapeutic Process.»Psychological Science 8 (3):162-166. doi: 10.1111/j.1467-9280.1997.tb00403.x. 
Petko, Dominik. 2013. «Lerntagebuch schreiben mit Weblogs. Didaktische Grundlagen und technische Entwicklungen am Beispiel von lerntagebuch.ch.» In E-Portfolio an der Schnittstelle von Studium und Beruf, herausgegeben von Damian Miller und Benno Volk, 206-214. Münster: Waxmann.

Petko, Dominik, Nives Egger, und Marc Graber. 2014. «Supporting learning with weblogs in science education: A comparison of blogging and hand-written reflective writing with and without prompts.» Themes in Science and Technology Education 7 (1):3-17.

Petko, Dominik, Nives Egger, Felix M. Schmitz, Alexandra Totter, Thomas Hermann, und Sissel Guttormsen. 2015. «Coping through blogging: A review of studies on the potential benefits of weblogs for stress reduction.» Cyberpsychology: Journal of Psychosocial Research on Cyberspace 9 (2), article 5. doi:10.5817/CP2015-2-5.

Ryan, Richard M., und Edward L. Deci. 2000. "Self-determination theory and the facilitation of intrinsic motivation, social development, and well-being». American psychologist 55 (1):68-78. doi:10.1037/0003-066X.55.1.68.

Sim, Jeffrey W. S., und Khe Foon Hew. 2010. «The use of weblogs in higher education settings: A review of empirical research.» Educational Research Review 5 (2):151-163. doi:10.1016/j. edurev.2010.01.001.

Totter, Alexandra, Nives Egger, Felix Schmitz, Thomas Hermann, und Dominik Petko. 2016. «Online-Reflexion mittels Weblogs: Ein Mittel zur Auseinandersetzung mit belastenden Situationen in der berufspraktischen Ausbildung von Lehrpersonen?» Beiträge zur Lehrerinnen- und Lehrerbildung, 34 (2), 203-211.

Venkatesh, Viswanath, Michael G. Morris, Gordon B. Davis, und Fred D. Davis. 2003. «User acceptance of information technology: Toward a unified view». MIS quarterly 27 (3): 425-478. http://www.jstor.org/stable/30036540.

Venkatesh, Viswanath, James Y. L. Thong, und Xin Xu. 2012. «Consumer acceptance and use of information technology: extending the unified theory of acceptance and use of technology.» MIS quarterly 36 (1):157-178.

Wopereis, Iwan G. J. H., Peter B. Sloep, und Sybilla H. Poortman. 2010. «Weblogs as instruments for reflection on action in teacher education.» Interactive Learning Environments 18 (3):245-261. doi:10.1080/10494820.2010.500530.

Xie, Ying, Fengfeng Ke, und Priya Sharma. 2008. «The effect of peer feedback for blogging on college students' reflective learning processes.» Internet and Higher Education 11 (1):1825. doi:10.1016/j.iheduc.2007.11.001.

\section{Abbildungen}

Abb. 1.: Nützlichkeit von praktikumsbegleitenden Weblogs im Vergleich zwischen Studierenden mit $(n=36)$ und ohne Peerfeedback ( $n=38)$.

Abb. 2.: Motivation der Studierenden zum Einsatz von praktikumsbegleitenden Weblogs im Vergleich zwischen Studierenden mit $(n=36)$ und ohne Peerfeedback $(n=38)$.

Abb. 3.: Einstellung zum Einsatz von praktikumsbegleitenden Weblogs im Vergleich zwischen Studierenden mit ( $n=36)$ und ohne Peerfeedback $(n=38)$. 